\title{
Percepciones de los estudiantes sobre el sistema universitario en el marco del Espacio Europeo de Educación Superior: participación en programas de movilidad internacional
}

Student perception about the university system in the context of European Higher Education: participation in international mobility programs

\author{
M. $^{a}$ Del Carmen Pegalajar Palomino ${ }^{1}$ \\ mcpegala@ujaen.es \\ Eufrasio Pérez Navío \\ epnavio@ujaen.es \\ M. a Jesús Colmenero Ruiz \\ mjruiz@ujaen.es \\ Universidad de Jaén, España
}

\section{Resumen:}

Este trabajo pretende conocer las percepciones del alumnado del Grado de Primaria de la Universidad de Jaén acerca del sistema universitario, prestando especial atención al Espacio Europeo de Educación Superior (EEES) y la movilidad internacional de los estudiantes. Para ello, se utiliza una metodología descriptiva, explorando mediante la técnica de la encuesta a través de un cuestionario ad hoc $(\mathrm{n}=230)$. Los resultados revelan la opinión favorable del alumnado hacia la planificación y organización docente, a pesar de mostrar su indiferencia ante las titulaciones académicas

\begin{abstract}
:
This paper aims to understand the perceptions of Primary Education Degree students at the University of Jaén about the university system, paying particular attention to the European Higher Education Area (EHEA) and the international mobility of students. To do this, the authors use a descriptive methodology, using an ad hoc questionnaire $(n=230)$. The results show the favorable opinion of students towards teaching planning and organization, despite showing his indifference to degrees and curricula, educational services provided from the university, students' learning
\end{abstract}

1 Dirección para correspondencia (Correspondence address):

María Del Carmen Pegalajar Palomino. Dpto. de Pedagogía. Facultad de Humanidades y Ciencias de la Educación. Universidad de Jaén. Paraje "Las Lagunillas", s/n. 23071 Jaén (España). 
Percepciones de los estudiantes sobre el sistema universitario en el marco del Espacio Europeo de Educación Superior: participación en programas de movilidad internacional M. ${ }^{a}$ del Carmen Pegalajar Palomino, Eufrasio Pérez Navío y M. ${ }^{a}$ Jesús Colmenero Ruiz

y planes de estudio, atención educativa prestada desde la universidad, proceso de aprendizaje del alumnado y conocimiento académico sobre el Grado. Además, se demuestra la existencia de diferencias estadísticamente significativas en las percepciones del alumnado sobre el sistema universitario según éstos hayan participado en programas de movilidad internacional; concretamente, hacia aspectos referidos a la planificación, organización docente y conocimiento académico sobre el Grado, siendo más favorables para alumnado que ha visitado alguna universidad europea.

\section{Palabras clave:}

Espacio Europeo de Educación Superior; alumnado; programa Erasmus; Grado de Educación Primaria. process, and academic knowledge on the Degree studied. Furthermore, it demonstrates the existence of statistically significant differences in the perceptions of students on the university system according to whether or not they have participated in international mobility programs. These differences are especially obvious in matters relating to planning, teaching and academic knowledge about the Degreestudents who have studied in a foreign university have a more favorable opinion about these aspects than those who have not.

\section{Key words:}

European Higher Education Area; students; Erasmus Program; Early Education Degree; Elementary Education Degree.

\section{Résumé:}

Cet article vise à comprendre les perceptions des futurs enseignants de primaire à I'Université de Jaén sur le système universitaire, en accordant une attention particulière à l'Espace européen de l'enseignement supérieur (EEES) et la mobilité internationale des étudiants. Pour ce faire, une méthode descriptive a été utilisée, explorant à travers la technique de sondage par le biais d'un questionnaire ad hoc $(n=230)$. Les résultats montrent l'avis favorable des étudiants vers la planification et l'organisation pédagogique, en dépit de montrer son indifférence à l'égard des services éducatifs, universitaires et de programmes fournis par l'université, le processus d'apprentissage des élèves et les connaissances académiques sur le niveau. En outre, il démontre l'existence de différences statistiquement significatives dans la perception des étudiants sur le système universitaire où ils ont participé à des programmes de mobilité internationale, en particulier, aux questions relatives à la planification, l'enseignement et l'organisation des connaissances théoriques sur le niveau, étant plus favorable pour les étudiants qui ont visité une université européenne.

\section{Mots clés:}

Espace européen de l'enseignement supérieur; étudiants; Erasmus; degré de lıenseignement primaire.

Fecha de recepción: 30-5-2013

Fecha de aceptación: 22-1-2014

\section{Introducción}

El proceso de convergencia europea es ya una realidad en la vida universitaria española. La Declaración de Bolonia (como se ha denominado al proceso de cambio y adaptación en el que estamos inmersos) constituye el punto de partida y la guía del proceso de convergencia la cual pretende (García y Pérez, 2008): 
- Adoptar un catálogo de titulaciones fácilmente legible y comparable que permita el reconocimiento e identidad común en el ámbito educativo europeo mediante la implementación, por ejemplo, del Suplemento Europeo al Título.

- Estructurar los estudios universitarios en dos ciclos (Grado y Postgrado) armonizando en torno al nuevo planteamiento de títulos, contenidos curriculares y materias, de tal forma que el título otorgado al terminar el primer ciclo tenga valor específico en el mercado de trabajo y el segundo ciclo conduzca a la obtención de un Máster o Doctorado.

- Implantación de un sistema de créditos ECTS que facilite la movilidad de los estudiantes y el reconocimiento de titulaciones. El sistema europeo de transferencia y acumulación de créditos (ECTS) es un sistema de valoración que parte del aprendizaje del alumno y que se basa en el desarrollo del trabajo necesario para lograr los objetivos de un programa formativo definidos a través de competencias adquiridas y resultados de aprendizaje.

- Reformular la educación en torno al aprendizaje del alumno y no en la enseñanza articulada desde la transmisión de contenidos, apostando por la garantía de calidad en criterios cooperativos de evaluación y metodología docente, logrando el desarrollo de la autonomía en el proceso de aprendizaje de los alumnos.

- Reconocer y fortalecer la movilidad de estudiantes, profesores y personal de administración y servicios así como alentar la competitividad en investigación y formación continua a lo largo de la vida.

- Formular criterios académicos, competenciales y de cualificación profesional que respondan a la demanda del mercado laboral europeo y que permitan responder a los retos educativo, laborales y sociales actuales.

Esta reforma ha supuesto no sólo un profundo cambio de tipo estructural, centrado en adecuar determinados rasgos formales comunes a todas las instituciones de Educación Superior (Jacobs y Van der Ploeg, 2006) sino, también, en la adopción de un enfoque diferente de docencia (Tomusk, 2006; Escorcia, Gutiérrez y Henríquez, 2007; Herrera y Enrique, 2008).

En este contexto, la principal función del profesor universitario consiste en posibilitar, facilitar y guiar al alumno para que pueda acceder 
intelectualmente a los contenidos y prácticas profesionales de una determinada disciplina (Herrera, 2007; Moreno et al., 2007; Sander, 2005). Para ello, se requiere de un sistema de aprendizaje autónomo y tutorizado que lleve al estudiante a construir e interpretar el entorno de manera significativa (Herrera y Cabo, 2008; Gairín et al., 2004) mediante un aprendizaje a lo largo de toda la vida (Herrera, Lorenzo y Rodríguez, 2008; Méndez, 2005). Además, se plantea un profundo cambio de paradigma educativo, centrando la educación del estudiante en las competencias que debe desarrollar y en los procesos de adquisición y construcción de ese conocimiento (Michavila y Esteve, 2011) para su formación integral e incorporación al mundo del trabajo (Gil, 2012). No obstante, Rodríguez $(2009,159)$ afirma cómo "el éxito o fracaso de las innovaciones educativas depende, en gran parte, de la forma en que los diferentes actores educativos interpretan, redefinen, filtran y dan forma a los cambios propuestos".

El informe "Student Centred Learning: Time for a New Paradigm in Education" (ESU, 2010) evidencia con claridad cómo esta reforma no sólo exige de una actualización de los planes de estudio, sino que plantea la necesidad de que la institución desarrolle su propio modelo educativo desde el que impulsar este cambio de paradigma. Ello ha obligado a las universidades a plantearse el nivel de adecuación del modelo educativo de su institución y, en caso contrario, a analizar la posibilidad de diseñar e implementar uno de nueva creación para poder afrontar los principios de esta reforma con garantías de calidad (Froment et al., 2010).

Para ello, tal y como se ha señalado anteriormente, se busca y promueve de manera esencial la movilidad de los estudiantes y profesores. Los programas de movilidad constituyen, de este modo, una línea fundamental de actuación para facilitar el intercambio de contenidos, metodologías y recursos y para potenciar la generación y transmisión de conocimiento (Pineda, Moreno y Belvis, 2008). Así pues, el programa Erasmus es considerado uno de los grandes impulsores de la movilidad académica europea en el nivel de la enseñanza superior durante los últimos 25 años El crecimiento de la movilidad de estudiantes en nuestro país ha sido constante a lo largo de estos años. España siempre ha ocupado uno de los primeros puestos entre los países europeos en lo que a movilidad de estudiantes se refiere, siendo desde el curso 2001/02 el primer país receptor de estudiantes y desde el curso 2009/10 también el 
Percepciones de los estudiantes sobre el sistema universitario en el marco del Espacio Europeo de Educación Superior: participación en programas de movilidad internacional

M. a del Carmen Pegalajar Palomino, Eufrasio Pérez Navío y M. ${ }^{a}$ Jesús Colmenero Ruiz

primer país emisor. De hecho, según los últimos datos disponibles, en el curso 2010/11 España recibió 37.433 estudiantes europeos y envió 36.183 universitarios (INE, 2012). En este sentido, Figel (2006, 2), Comisario europeo de Educación, Formación, Cultura y Multilingüismo, ha señalado cómo "Erasmus ha sido y sigue siendo un factor clave en la internacionalización y, de alguna manera, de la "europeización" de los sistemas de educación superior de la Unión Europea". El éxito del programa Erasmus ha propiciado la creación de otras experiencias y redes de movilidad nacionales como el programa SICUE, que potencia la movilidad entre universidades españolas desde el año 2000.

La investigación desarrollada por Carrera, Bonete y Muñoz (2007) describe el perfil del alumno español que opta a un programa Erasmus siendo éste: mujer, de edad media en torno a 22 años y la estancia media en 6.5 meses, muy ligeramente por encima de la media europea. Por su parte, el trabajo de Brakel et al. (2004) evalúa los impactos institucionales y nacionales del programa, entre los que se destaca la importancia de la atención institucional prestada al reconocimiento de cualificaciones a través de los créditos europeos así como el aprendizaje de las lenguas. Del mismo modo, Bracht et al. (2006) confirma cómo el hecho de participar en un programa Erasmus puede constituir una ventaja clave para encontrar trabajo ya que se considera que realizar estudios en el extranjero es una experiencia valiosa que abre puertas para los alumnos. Por su parte, el estudio de la Tempus Public Foundation (2006) "Dissemination of results and best practices for raising the profile of Erasmus Mobility" destaca la importancia de la enseñanza de los idiomas y de la cultura del país receptor como instrumento de promoción de las instituciones superiores y los propios países.

Centrados en la valoración general del alumnado acerca del EEES, el trabajo de Méndez (2008) ha revelado cómo la información que manejan los estudiantes sobre la universidad, sobre su rol como universitarios y sobre el EEES no es suficiente, lo que repercute de forma negativa en que este colectivo pueda adoptar el papel que se le atribuye dentro del proceso de convergencia europea. La investigación desarrollada por Font-Mayolas y Masferrer (2010) ha destacado el elevado número de estudiantes que declaran no disponer de suficiente información sobre el EEES, añadiendo que sólo reciben información negativa y a veces distorsionada, aunque al mismo tiempo, puede haber faltado interés por su parte en documentarse. Más recientemente, Coterón, Franco y Gil 
(2012) han concluido cómo el alumnado no valora como positivos los cambios introducidos por el nuevo modelo europeo, mostrándose más satisfechos los estudiantes de Grado que los de Licenciatura. De igual modo, Leo y Cubo (2012) han observado cómo el alumnado considera que no ha recibido información suficiente sobre la adaptación al EEES para llevar a cabo una incorporación eficaz: no conocen el número de horas correspondientes a un crédito europeo, desconocen las posibilidades de movilidad y opinan que la universidad no posee los recursos adecuados para una adaptación de calidad.

Sin embargo, Otero, Ferro y Vila (2012) han puesto de manifiesto cómo el nuevo marco europeo de Educación Superior mejora el grado de satisfacción de los alumnos respecto al profesorado y la aplicación de los conocimientos. Por su parte, el estudio realizado por Edwards, Donderis y Ballester (2005) revela una escasa implicación de profesores y estudiantes en el proceso, evidenciándose la necesidad de implementar acciones específicas de sensibilización y formación para provocar el necesario cambio de cultura académica y así poder avanzar con éxito hacia la convergencia europea en el sistema universitario español. Más concretamente, centrados en la repercusión que el proceso de convergencia europea puede tener sobre el sistema universitario español, se subraya el trabajo de Castaño et al. (2007) el cual ha analizado el impacto de la puesta en marcha de un plan de convergencia, destacando un aumento en la valoración de la labor del profesorado, a pesar de destacar deficiencias como la coordinación docente y el seguimiento del aprendizaje del alumnado.

\section{Objetivos y metodología}

Con este trabajo se pretende conocer las percepciones del alumnado de tercer curso del Grado de Educación Primaria de la Universidad de Jaén sobre la el sistema universitario, prestando especial importancia hacia el proceso de convergencia europea. De igual modo, se analiza si el hecho de que el estudiante haya participado en un programa de movilidad internacional influye en las valoraciones que éste pueda tener sobre la universidad.

Para ello, se ha utilizado un estudio descriptivo basado en la técnica de la encuesta a través de un cuestionario ad hoc denominado "Cuestionario sobre las percepciones de los estudiantes hacia el sistema universitario". Se trata de una escala tipo Likert cuyas respuestas oscilan entre 
Percepciones de los estudiantes sobre el sistema universitario en el marco del Espacio Europeo de Educación Superior: participación en programas de movilidad internacional

M. a del Carmen Pegalajar Palomino, Eufrasio Pérez Navío y M. ${ }^{a}$ Jesús Colmenero Ruiz

1 y 5 (siendo $1=$ plenamente de acuerdo y $5=$ totalmente en desacuerdo) compuesta por seis dimensiones fundamentales: 1) Planificación docente, referida a la valoración general del alumnado acerca de la actividad docente; 2) Titulaciones académicas y planes de estudio, analiza las potencialidades de los planes de estudio para el aprendizaje del alumnado universitario; 3) Organización Docente, representada por cuestiones de planificación y desarrollo para la mejora del proceso de enseñanza y aprendizaje en la Educación Superior; 4) Atención educativa desde la Universidad, referida a la estimación del estudiante acerca de la propia institución de Educación Superior; 5) Proceso de aprendizaje del alumno en Educación Superior, centrado en el análisis de elementos favorecedores de un proceso de enseñanza universitaria de calidad; 6) Conocimiento académico sobre el Grado, dónde se recoge el grado de información que posee el estudiante acerca de la titulación que cursa.

Construida la escala, se procedió a la validación por parte de expertos universitarios en el área de Didáctica y Organización Escolar (DOE) a los que se les pidió que evaluaran y validaran la pertinencia de cada ítem del cuestionario, estableciendo las consideraciones oportunas para su mejora en cuanto a su contenido, pertinencia, ambigüedad, redacción y otros aspectos. Una vez estas sugerencias fueron incorporadas al instrumento, se procedió a la aplicación de una prueba piloto a cincuenta estudiantes de tercer curso del Grado de Educación Primaria de la Escuela de Magisterio "Sagrada Familia" de Úbeda, centro adscrito a la propia Universidad de Jaén aunque de titularidad concertada. Esta prueba tenía como intención analizar la validez de constructo; así, el índice de adecuación muestral KMO alcanza un valor de .890 y la prueba de esfericidad de Bartlett es de 4875.251 ( $p=.000)$. Estos datos hacen que se rechace la hipótesis nula de que la matriz de correlación inter-ítems es identidad y se consideran que las respuestas están sustancialmente relacionadas. Por su parte, el análisis de los componentes principales revela, tras la rotación Varimax, la convergencia de seis factores que explican el $52.665 \%$ de la varianza; los ítems muestran valores apropiados, situándose entre .459 y .786 .

El estudio de la fiabilidad confirma la alta consistencia interna del instrumento al alcanzar el coeficiente Alfa de Cronbach el valor de .933, lo que indica que la escala diseñada es muy confiable. Por su parte, para el método de las dos mitades se obtiene, para la primera parte un valor de .890 y para la segunda un valor de .891, lo que vuelve a corroborar los resultados obtenidos en el procedimiento anterior. 
La población objeto de estudio está compuesta por el alumnado del tercer curso del Grado de Educación Primaria de la Universidad de Jaén. En total, la población asciende a 234 estudiantes, estando compuesta la muestra por 230 alumnos universitarios que participaron en la investigación cumplimentando el cuestionario proporcionado. Para la selección de los sujetos se ha utilizado un muestreo probabilístico aleatorio simple (Tójar y Matas, 2009), de forma que se han ido adscribiendo a la muestra todos aquellos sujetos que al azar respondieron a los cuestionarios enviados. Para el cálculo de la muestra participante se ha utilizado la fórmula para poblaciones con menos de 100.000 sujetos con un nivel de confianza del $95 \%$ y un error de estimación máximo de un 4\%.

En la investigación han participado, en cuanto al género, más mujeres que hombres $(60.0 \%$ y $40.0 \%$, respectivamente) cuyas edades oscilan, de manera mayoritaria, entre los 20 y 25 años (90.9\%). En cuanto a la forma de acceso a la Universidad, la inmensa mayoría del alumnado procede de Bachillerato, diferenciándose tan sólo por el itinerario elegido $(67.0 \%$ en Humanidades y Ciencias Sociales, $19.1 \%$ para Ciencias y Tecnología y $0.9 \%$ en Arte). Finalmente, y considerando su participación en programas de movilidad internacional, se destaca cómo una abrumadora mayoría (97.4\%) no ha realizado estudios en otras universidades europeas dentro del programa Erasmus.

\section{Resultados}

Con la intención de analizar las valoraciones del alumnado del Grado de Educación Primaria de la Universidad de Jaén sobre el sistema universitario, se han examinado las puntuaciones de las medias y desviaciones típicas obtenidas para cada uno de los ítems de los diferentes factores que componen la escala.

\section{Planificación Docente}

La tabla $\mathrm{n}^{\circ} 1$ refleja las puntuaciones medias obtenidas en este primer factor, las cuáles revelan cómo el alumnado encuestado se muestra conforme con la utilización, por parte del profesorado, de espacios virtuales para difundir materiales y fomentar la enseñanza virtual $(M=2.23$; D.T.=1.099). Del mismo modo, considera que existe coherencia entre 
Percepciones de los estudiantes sobre el sistema universitario en el marco del Espacio Europeo de Educación Superior: participación en programas de movilidad internacional

M. a del Carmen Pegalajar Palomino, Eufrasio Pérez Navío y M. ${ }^{a}$ Jesús Colmenero Ruiz

los contenidos teóricos y las prácticas desarrolladas en las distintas asignaturas $(M=2.65$; D.T.=1.074), estableciéndose relaciones con los alumnos basadas en un clima empático ( $M=2.69$; D.T.=0.997); asume que se ponen a disposición del alumnado suficientes y adecuados recursos materiales $(M=2.76$; D.T.=0.975) a la vez que sostiene cómo las tutorías facilitan la comprensión y asimilación de contenidos ( $M=2.78$; D.T.=0.997). En general, el alumnado manifiesta que el profesorado de su titulación da respuesta a sus necesidades académicas $(M=2.89$; D.T. $=1.011)$ planteando, para ello, diferentes tipos de actividades $(M=2.82$; D.T.=1.016) así como un sistema de evaluación capaz de contribuir al desarrollo del aprendizaje $(M=2.89 ;$ D.T.=0.921). En cuanto a las estrategias metodológicas, los estudiantes se muestran indiferentes hacia el hecho de que éstas se adecúen a sus características individuales ( $M=2.89$; D.T.=1.000) así como el hecho de que el docente haga uso de bibliografía actualizada y de fácil acceso ( $M=2.90$; D.T.=1.123).

Tabla 1. Planificación docente

\begin{tabular}{|c|c|c|}
\hline & M. & D.T. \\
\hline $\begin{array}{l}\text { 39. El profesorado utiliza espacios virtuales para difundir mate- } \\
\text { riales y fomentar la enseñanza virtual }\end{array}$ & 2.23 & 1.099 \\
\hline $\begin{array}{l}\text { 43. En general, existe coherencia entre los contenidos teóricos y } \\
\text { las prácticas de las asignaturas desarrolladas en el aula }\end{array}$ & 2.65 & 1.074 \\
\hline $\begin{array}{l}\text { 41. La relación profesor-alumno permite crear un clima empáti- } \\
\text { co para favorecer el proceso de enseñanza-aprendizaje }\end{array}$ & 2.69 & 0.997 \\
\hline $\begin{array}{l}\text { 37. Los recursos materiales de los que dispone el profesor para } \\
\text { la impartición de su asignatura son suficientes y adecuados }\end{array}$ & 2.76 & 0.975 \\
\hline $\begin{array}{l}\text { 42. Las tutorías han facilitado la comprensión y asimilación de } \\
\text { contenidos propios de mi titulación }\end{array}$ & 2.78 & 0.997 \\
\hline $\begin{array}{l}\text { 44. Percibo que el profesorado de mi titulación da respuesta a } \\
\text { las necesidades educativas del alumnado }\end{array}$ & 2.80 & 1.011 \\
\hline $\begin{array}{l}\text { 36. En el aula, el profesorado plantea diferentes tipos de activi- } \\
\text { dades para una mejor adquisición de las competencias }\end{array}$ & 2.82 & 1.016 \\
\hline $\begin{array}{l}\text { 40. El sistema de evaluación utilizado ha contribuido al desarro- } \\
\text { Ilo de mi aprendizaje }\end{array}$ & 2.89 & .921 \\
\hline $\begin{array}{l}\text { 35. La metodología empleada en las clases por el profesor se } \\
\text { adecúa a las características del alumnado }\end{array}$ & 2.89 & 1.000 \\
\hline $\begin{array}{l}\text { 38. En general, el profesorado utiliza referencias bibliográficas } \\
\text { actuales y de fácil acceso }\end{array}$ & 2.90 & 1.123 \\
\hline
\end{tabular}




\section{Titulaciones académicas y planes de estudio}

Según se muestra en la tabla n², el alumnado encuestado sostiene cómo el proceso de convergencia europea favorece el trabajo autónomo del estudiante $(M=2.69 ;$ D.T.=1.073), fomenta la participación activa del alumnado en el aula $(M=2.72 ;$ D.T.=1.020), contribuye al aprendizaje de una lengua extranjera $(M=2.73$; D.T.=1.196) así como también mejora la formación en competencias ( $M=2.77$; D.T.=1.018) y la homogeneización de los estudios universitarios en el contexto europeo $(M=2.80$; D.T.=1.051).

Tabla 2. Titulaciones académicas y planes de estudio

M. D.T.

5. Los nuevos planes de estudio favorecen el trabajo autónomo del estudiante universitario

8. La nueva normativa europea fomenta la participación activa del alumnado universitario en el aula

6. La nueva normativa europea en educación universitaria ha contribuido al aprendizaje de una lengua extranjera en el alum- 2.731 .196 nado universitario

2. Los nuevos planes de estudios mejoran la formación en competencias del alumnado universitario

1. Considero que la adaptación de los planes de estudio al contexto europeo favorece la homogeneización de estudios universitarios

3. Los nuevos planes de estudio contribuyen a la formación y al desarrollo profesional de los estudiantes de cara a la inserción socio-laboral

7. La nueva normativa europea ha posibilitado la movilidad de los estudiantes en las universidades

4. La nueva reestructuración de las titulaciones en Grado y Máster mejora la calidad de la educación universitaria

28 .Considero que los nuevos planes de estudio permiten una mayor especialización en mi titulación

27. Considero que las menciones establecidas en el Grado que estoy cursando son suficientes y adecuadas

Sin embargo, muestra su total indiferencia al considerar cómo los nuevos planes de estudio contribuyen a la formación y desarrollo profesional 
de los mismos ( $M=2.98$; D.T.=1.082) y posibilitan la movilidad de los estudiantes $(M=3.01$; D.T.=1.090). Del mismo modo, se muestran poco favorables al determinar cómo la nueva reestructuración de las titulaciones ha mejorado la calidad de la educación universitaria ( $M=3.02$; D.T.=1.096), permite una mayor especialización $(M=3.16$; D.T.=1.184) y considera suficientes y adecuadas las menciones establecidas para el Grado de Educación Primaria en la Universidad de Jaén ( $M=3.33$; D.T.=1.339).

\section{Organización académica}

Analizando las puntuaciones obtenidas en la tabla $n^{\circ} 3$, se destaca el acuerdo de los encuestados en recalcar la importancia para la formación académica del alumnado del desarrollo del Prácticum $(M=2.15$; D.T.=1.398), subrayando la figura del tutor para que exista conexión entre el centro educativo y la propia institución de Educación Superior $((M=2.23 ;$ D.T.=1.487). Del mismo modo, consideran que debería reducirse la ratio de alumnos por aula $(M=2.29$; D.T.=1.199) sosteniendo cómo la asistencia y participación del alumnado en las clases contribuye a la mejora del proceso de enseñanza-aprendizaje ( $M=2.33$; D.T.=1.047), adaptándose a sus necesidades las instalaciones ofrecidas por la Universidad ( $M=2.60 ;$ D.T.=1.116). No obstante, muestran su indiferencia ante el hecho de que las asignaturas impartidas entre varios profesores facilita el proceso de enseñanza-aprendizaje ( $M=3.86$; D.T.=1.225).

Tabla 3. Organización académica

M. D.T.

24. El desarrollo del Prácticum complementa mi formación académica

2.151 .398

25. Considero fundamental la figura del tutor de Prácticum para que haya una conexión entre el centro educativo y la universidad

34. Considero que debería de reducirse el número de alumnos por aula

33. La asistencia y participación del alumnado contribuye a la mejora del proceso de aprendizaje

18. Las instalaciones de la Universidad para la impartición de las clases son adecuadas y se adaptan a mis necesidades

20. Las asignaturas impartidas entre varios profesores facilita el proceso de enseñanza-aprendizaje 


\section{Atención educativa desde la Universidad}

Para este factor, tal y como se muestra en la tabla $\mathrm{n}^{\circ} 4$, las puntuaciones medias demuestran cómo los alumnos encuestados se muestran poco favorables con el profesorado de su titulación al considerar que éste no cumple con sus expectativas formativas ( $M=2.89$; D.T.=0.972); asimismo, su valoración tampoco es positiva hacia la labor desempeñada por la Facultad de Humanidades y Ciencias de la Educación de la Universidad $(M=2.96$; D.T.=1.029), la enseñanza recibida en el Grado que están cursando $(M=2.96$; D.T. $=1.044)$ así como su apreciación sobre la Universidad $(M=3.07$; D.T.=1.129). Consideran que desde la propia institución no se les ha informado adecuadamente acerca de los nuevos planes de estudio y sus implicaciones para sus estudios universitarios $(M=3.20$; D.T.=1.233) a la vez que realizan una evaluación poco favorable hacia el nuevo plan de estudios ( $M=3.30 ;$ D.T.=1.113).

Tabla 4. Atención educativa desde la Universidad

\begin{tabular}{|c|c|c|}
\hline & M. & D.T. \\
\hline $\begin{array}{l}\text { 32. En líneas generales, el profesorado de la titulación cumple } \\
\text { con mis expectativas formativas }\end{array}$ & 2.89 & 0.972 \\
\hline $\begin{array}{l}\text { 30. La Facultad desempeña una labor importante en mi plan de } \\
\text { estudios }\end{array}$ & 2.96 & 1.029 \\
\hline 31. La enseñanza recibida en mi titulación es de calidad & 2.96 & 1.044 \\
\hline $\begin{array}{l}\text { 29. En líneas generales, mi valoración sobre la Universidad es } \\
\text { positiva }\end{array}$ & 3.07 & 1.129 \\
\hline $\begin{array}{l}\text { 10. Desde la Universidad (profesorado, administrativo...) se } \\
\text { me ha informado acerca de los nuevos planes de estudio y sus } \\
\text { implicaciones para mis estudios }\end{array}$ & 3.20 & 1.233 \\
\hline $\begin{array}{l}\text { 12. Mi valoración acerca del nuevo plan de estudios de mi titu- } \\
\text { lación es positiva }\end{array}$ & 3.30 & 1.113 \\
\hline
\end{tabular}

\section{Proceso de aprendizaje del alumno en Educación Superior}

En este factor, la tabla $\mathrm{n}^{\circ} 5$ demuestra cómo los estudiantes se muestran poco convencidos acerca de la adecuación del número de convocatorias establecidas por la Universidad para el desarrollo de los exámenes $(M=2.98$; D.T.=1.285). A su vez, se muestran indiferentes al considerar 
que el Plan de Acción Tutorial se adecúa a sus necesidades $(M=3.15$; D.T.=1.080) así como que la distribución del nuevo plan de estudios les permite desarrollar un proceso de enseñanza de calidad $(M=3.26$; D.T.=0.967). De igual modo, no se muestran muy favorables acerca de si el horario de clases les facilita el aprendizaje $(M=3.45$; D.T.=1.206) y la adecuación del calendario de exámenes a sus intereses y necesidades académicas $(M=3.47$; D.T.=1.256).

Tabla 5. Proceso de aprendizaje del alumno en Educación Superior

\begin{tabular}{lrr}
\hline & M. & D.T. \\
\hline $\begin{array}{l}\text { 17. El número de convocatorias de los exámenes de las asigna- } \\
\text { turas de mi titulación (ordinarias y extraordinarias) es suficiente }\end{array}$ & 2.98 & 1.285 \\
$\begin{array}{l}\text { 23. El Plan de Acción Tutorial de mi titulación se adecúa a mis } \\
\text { necesidades }\end{array}$ & 3.15 & 1.080 \\
$\begin{array}{l}\text { 15. La distribución del plan de estudios de mi titulación permite } \\
\text { desarrollar un proceso de enseñanza de calidad }\end{array}$ & 3.26 & 0.967 \\
$\begin{array}{l}\text { 14. El horario de clases facilita el aprendizaje del alumnado } \\
\text { universitario }\end{array}$ & 3.45 & 1.206 \\
$\begin{array}{l}\text { 16. El calendario de exámenes se adecúa a mis intereses y ne- } \\
\text { cesidades académicas y funcionalidad en las actividades de la } \\
\text { vida diaria }\end{array}$ & 3.47 & 1.256 \\
\hline
\end{tabular}

\section{Conocimiento académico sobre el Grado}

Para este último factor, tal y como se muestra en la tabla $n^{\circ}$ 6, el alumnado encuestado considera que conoce las menciones de establecidas para el Grado de Educación Primaria $(M=2.48$; D.T.=1.369) y que las guías docentes les permite un mayor conocimiento de las asignaturas $(M=2.68$; D.T. $=1.082)$. Asimismo, manifiesta conocer tanto las competencias ( $M=2.80$; D.T. $=1.041)$ como los resultados de aprendizaje $(M=2.89$; D.T.=.978) del Grado que está cursando. Sin embargo, muestran su indiferencia sobre la suficiencia de la información recibida acerca de los aspectos académicos de su titulación ( $M=3.04$; D.T.=1.075), la información recibida para los nuevos planes de estudio por medios ajenos a la propia institución de Educación Superior $(M=3.11$; D.T.=1.213) y, finalmente, afirman mostrarse poco convencidos acerca del conocimiento que poseen sobre el sistema de créditos europeos ( $M=3.28$; D.T.=1.156). 
Tabla 6. Conocimiento académico sobre el Grado

M. D.T.

26. Conozco las diferentes menciones establecidas por la Universidad en el Grado que estoy cursando

19 Las guías docentes me permiten un mayor conocimiento de las asignaturas

21. Conozco las competencias del Grado que estoy cursando

22. Conozco los resultados de aprendizaje del Grado que estoy cursando

13. Dispongo de suficiente información sobre los aspectos académicos de mi titulación

9. He recibido informaciones acerca de los nuevos planes de estudio de la educación superior por cualquier medio ajeno a la $3.11 \quad 1.213$ universidad (radio, prensa, Internet...)

11. Conozco íntegramente el nuevo sistema de créditos (Sistema Europeo de Transferencia de Créditos, ECTS)

\section{Diferencias de medias según la participación de los estudiantes en programas de movilidad internacional}

Tomando como referencia los factores anteriormente mencionados y la variable cualitativa "participación en programas de movilidad", se constatan diferencias estadísticamente significativas en algunos de los ítems tras aplicar la prueba T de Student. En este caso, tan sólo se han comentado aquellos factores en los que alguno de sus ítems eran significativos y, por tanto, se apreciaban diferencias estadísticas.

Más concretamente, dichas diferencias se sitúan entre el alumnado que ha participado en un programa de movilidad y por lo tanto, ha tenido la oportunidad de viajar al extranjero y cursa parte de sus estudios en alguna universidad europea y aquellos que no lo han hecho; en este caso, las significaciones se muestran más favorables para aquellos alumnos que se han acogido a un programa de movilidad internacional.

Así pues, tal y como se muestra en la tabla $\mathrm{n}^{\circ} 7$, para el factor "Planificación docente" tan sólo se aprecian diferencias significativas en las valoraciones del alumnado acerca del uso de espacios virtuales por parte del profesor para difundir materiales y fomentar la enseñanza virtual ( $\mathrm{t}(228)=-.896, \mathrm{p}=.045)$ así como referencias bibliográficas actuales y de fácil acceso $(\mathrm{t}(228)=-2.003, \mathrm{p}=.046)$. Más concretamente, las diferen- 
cias se muestran más favorables para aquellos alumnos que han participado en algún programa de movilidad internacional, permitiéndoles continuar sus estudios en alguna otra universidad europea.

De este modo, no se puede afirmar la existencia de diferencias significativas en las valoraciones de los estudiantes encuestados sobre cuestiones referidas a la planificación docente tales como: coherencia entre contenidos teóricos y prácticos, desarrollo de un clima empático profesor-alumno, uso adecuado de material en el aula, tutorías, respuesta las necesidades educativas por parte del docente, planteamiento de actividades variadas y desarrollo de un sistema de evaluación y metodología adecuado.

Tabla 7. Diferencias de medias según la participación del alumnado en programas de movilidad internacional para el factor "Planificación docente"

\begin{tabular}{|c|c|c|c|}
\hline & Participa & No participa & Sig. \\
\hline $\begin{array}{l}\text { 39. El profesorado utiliza espacios virtuales } \\
\text { para difundir materiales y fomentar la ense- } \\
\text { ñanza virtual }\end{array}$ & 1.83 & 2.24 & *.045 \\
\hline $\begin{array}{l}\text { 43. En general, existe coherencia entre los } \\
\text { contenidos teóricos y las prácticas de las } \\
\text { asignaturas desarrolladas en el aula }\end{array}$ & 2.33 & 2.66 & .356 \\
\hline $\begin{array}{l}\text { 41. La relación profesor-alumno permite crear } \\
\text { un clima empático para favorecer el proceso } \\
\text { de enseñanza-aprendizaje }\end{array}$ & 2.50 & 2.69 & .467 \\
\hline $\begin{array}{l}\text { 37. Los recursos materiales de los que dis- } \\
\text { pone el profesor para la impartición de su } \\
\text { asignatura son suficientes y adecuados }\end{array}$ & 2.50 & 2.77 & .365 \\
\hline $\begin{array}{l}\text { 42. Las tutorías han facilitado la comprensión } \\
\text { y asimilación de contenidos propios de mi } \\
\text { titulación }\end{array}$ & 2.17 & 2.79 & .274 \\
\hline $\begin{array}{l}\text { 44. Percibo que el profesorado de mi titula- } \\
\text { ción da respuesta a las necesidades educati- } \\
\text { vas del alumnado }\end{array}$ & 2.50 & 2.81 & .956 \\
\hline $\begin{array}{l}\text { 36. En el aula, el profesorado plantea dife- } \\
\text { rentes tipos de actividades para una mejor } \\
\text { adquisición de las competencias }\end{array}$ & 2.83 & 2.82 & .205 \\
\hline $\begin{array}{l}\text { 40. El sistema de evaluación utilizado ha con- } \\
\text { tribuido al desarrollo de mi aprendizaje }\end{array}$ & 2.67 & 2.90 & .292 \\
\hline
\end{tabular}


35. La metodología empleada en las clases por el profesor se adecúa a las características del alumnado

$\begin{array}{lll}2.50 & 2.90 \quad .741\end{array}$

38. En general, el profesorado utiliza referencias bibliográficas actuales y de fácil acceso

$2.00 \quad 2.92 \quad * .046$

Opciones de respuesta: $1=$ plenamente de acuerdo; $2=$ de acuerdo; $3=$ indiferente; $4=$ en desacuerdo; $5=$ totalmente en desacuerdo

${ }^{*}=p<.05$

En segundo lugar, y centrados en aspectos referidos a la "Organización académica", se destacan diferencias estadísticamente significativas en las valoraciones de los estudiantes acerca de la universidad, según su participación en programas de movilidad (tabla $\mathrm{n}^{\circ}$ 8). Así, los estudiantes que han accedido a cursar sus estudios universitarios en otras universidades europeas, muestran unas percepciones más favorables hacia el Prácticum como complemento para su formación académica $(\mathrm{t}(228)=-$ $1.457, \mathrm{p}=.029)$, la asistencia y participación a clase del alumnado para la mejora del proceso de aprendizaje $(\mathrm{t}(228)=-1.189, \mathrm{p}=.029)$ y la adecuación de las instalaciones $(\mathrm{t}(228)=-2.091, \mathrm{p}=.043)$. Sin embargo, sostienen cómo la impartición de asignaturas por parte de varios profesores dificulta su proceso de enseñanza-aprendizaje $(\mathrm{t}(228)=.957, \mathrm{p}=.026)$.

Tabla 8. Diferencias de medias según la participación del alumnado en programas de movilidad internacional para el factor "Organización académica"

\begin{tabular}{llll}
\hline & Participa & No participa & Sig. \\
\hline $\begin{array}{l}\text { 24. El desarrollo del Prácticum complementa } \\
\text { mi formación académica }\end{array}$ & 1.33 & 2.17 & $* .029$ \\
$\begin{array}{l}\text { 25. Considero fundamental la figura del tutor } \\
\text { de Prácticum para que haya una conexión }\end{array}$ & 1.67 & 2.24 & .061 \\
entre el centro educativo y la universidad & & & \\
$\begin{array}{l}\text { 34. Considero que debería de reducirse el } \\
\text { número de alumnos por aula }\end{array}$ & 3.00 & 2.27 & .181 \\
$\begin{array}{l}\text { 33. La asistencia y participación del alum- } \\
\text { nado contribuye a la mejora del proceso de } \\
\text { aprendizaje }\end{array}$ & 1.83 & 2.35 & $* .029$ \\
$\begin{array}{l}\text { 18. Las instalaciones de la Universidad para } \\
\text { la impartición de las clases son adecuadas y }\end{array}$ & 1.67 & 2.63 & $* .043$ \\
se adaptan a mis necesidades & & &
\end{tabular}


Percepciones de los estudiantes sobre el sistema universitario en el marco del Espacio Europeo de Educación Superior: participación en programas de movilidad internacional M. a del Carmen Pegalajar Palomino, Eufrasio Pérez Navío y M. ${ }^{a}$ Jesús Colmenero Ruiz

20. Las asignaturas impartidas entre varios profesores facilita el proceso de enseñanzaaprendizaje

Opciones de respuesta: $1=$ plenamente de acuerdo; $2=$ de acuerdo; $3=$ indiferente; $4=e n$ desacuerdo; $5=$ totalmente en desacuerdo

${ }^{*}=p<.05$

Finalmente, como se expone en la tabla $\mathrm{n}^{\circ}$ 9, para el factor "Conocimiento académico sobre el Grado" los datos obtenidos revelan cómo existen diferencias estadísticamente significativas en las percepciones de los estudiantes universitarios acerca del uso de las guías docentes para un mayor conocimiento de las asignaturas $(\mathrm{t}(228)=-1.950, \mathrm{p}=.010)$ y en el conocimiento de las competencias del Grado que están cursando $(\mathrm{t}(228)=-1.929, \mathrm{p}=.032)$, siendo más favorables para aquellos que se han adherido a un programa de movilidad internacional para estudiantes universitarios. No obstante, no se aprecian diferencias en las percepciones de los estudiantes sobre su conocimiento de las diferentes menciones establecidas por la universidad, resultados de aprendizaje de las asignaturas, información sobre aspectos académicos, los nuevos planes de estudio, sistema de créditos, etc.

Tabla 9. Diferencias de medias según la participación del alumnado en programas de movilidad internacional para el factor "Conocimiento académico sobre el Grado"

\begin{tabular}{lccc}
\hline & Participa & No participa & Sig. \\
\hline $\begin{array}{l}\text { 26. Conozco las diferentes menciones esta- } \\
\text { blecidas por la universidad en el Grado que } \\
\text { estoy cursando }\end{array}$ & 2.00 & 2.49 & .557 \\
$\begin{array}{l}\text { 19 Las guías docentes me permiten un mayor } \\
\text { conocimiento de las asignaturas }\end{array}$ & 1.83 & 2.70 & $* .010$ \\
$\begin{array}{l}\text { 21. Conozco las competencias del Grado que } \\
\text { estoy cursando }\end{array}$ & 2.00 & 2.83 & $* .032$ \\
$\begin{array}{l}\text { 22. Conozco los resultados de aprendizaje } \\
\text { del Grado que estoy cursando }\end{array}$ & 2.00 & 2.91 & .064 \\
$\begin{array}{l}\text { 13. Dispongo de suficiente información sobre } \\
\text { los aspectos académicos de mi titulación }\end{array}$ & 1.83 & 3.07 & .219
\end{tabular}


9. He recibido informaciones acerca de los nuevos planes de estudio de la educación superior por cualquier medio ajeno a la universidad (radio, prensa, Internet...)

11. Conozco íntegramente el nuevo sistema de créditos (Sistema Europeo de Transferencia de Créditos, ECTS)

Opciones de respuesta: $1=$ plenamente de acuerdo; $2=$ de acuerdo; $3=$ indiferente; $4=e n$ desacuerdo; $5=$ totalmente en desacuerdo

$$
*=p<.05
$$

\section{Conclusiones}

Esta investigación ha analizado las percepciones de los estudiantes ante el sistema universitario marcado, a día de hoy, por el proceso de convergencia europea. Ello requiere de una indagación continuada en las comunidades de Educación Superior para así, poder detectar las reacciones que se suceden en la realidad; de este modo, se pueden establecer mapas de indicadores que reflejen los puntos fuertes, las debilidades o carencias en los distintos ámbitos organizativos y en las personas para actuar en consecuencia (Villar y De la Rosa, 2004; Sevillano, 2004; Álvarez et. al, 2005).

Así pues, y en cuanto a la planificación docente, los estudiantes se muestran conformes con la actuación del profesorado y, más concretamente, con el uso de espacios virtuales para el desarrollo de sus asignaturas, coherencia entre contenidos teóricos y prácticos, desarrollo de un clima empático profesor-alumno, adecuación de los recursos materiales y tutorías. Resulta, pues, un aspecto de vital importancia ya que la calidad de la educación en el sistema universitario depende, en gran medida, de la planificación realizada por el docente para el desarrollo de su actividad en el aula. No obstante, los estudiantes de tercer curso del Grado de Educación Primaria de la Universidad de Jaén se muestren indiferentes en sus valoraciones acerca de las actividades planteadas por el docente, sistema de evaluación utilizado, metodología y uso de referencias bibliográficas actualizadas para el desarrollo del temario en sus asignaturas.

En este caso, la indiferencia en sus valoraciones pueden traducirse en el malestar del estudiante sobre la actividad docente, lo que repercute de forma directa en el desarrollo del proceso de aprendizaje del alum- 
nado universitario. La planificación de la docencia, desde este punto de vista, se orienta hacia la puesta en práctica de un plan cuidadosamente deliberado y articulado. Ello supone una de las competencias básicas del ejercicio profesional del docente, capaz de situar la intervención educativa en un contexto más amplio que le da sentido (Plan de estudios y perfil profesional, número de créditos disponibles, características de los estudiantes, competencias a desarrollar, etc.).

Respecto a las titulaciones académicas y los planes de estudio, los estudiantes encuestados sostienen cómo desde la universidad se favorece el trabajo autónomo del estudiante, la participación activa del alumnado en el aula y el aprendizaje de una lengua extranjera, lo cual ayuda al estudiante a construir e interpretar el mundo de manera significativa (Herrera y Cabo, 2008). Sin embargo, no se muestran muy favorables con el hecho de que los planes de estudio contribuyan a la homogeneización de los estudios universitarios, formación y desarrollo profesional del estudiante para su inserción socio-laboral, movilidad de los estudiantes, mejora de la calidad del sistema universitario y posibilidades de especialización. Sin duda, un aspecto sobre el que se ha de reflexionar pues, dado el proceso de convergencia europea, desde el sistema universitario se ha de trabajar concienzudamente para poder facilitar la formación y posterior acceso al mercado laboral de los estudiantes, posibilitando sus relaciones con el marco internacional.

El alumnado participante en la investigación ha mostrado, además, su indiferencia hacia la atención educativa prestada desde la universidad; no se muestran muy satisfechos con la labor desarrollada por la institución y, además, por la enseñanza que están recibiendo. De igual modo, consideran que desde la universidad no han sido suficientemente informados acerca del proceso realizado y sus implicaciones para los estudios que están cursando. En cuanto a la adecuación del proceso de aprendizaje a sus necesidades e intereses académicos, los datos revelan su preocupación hacia el número de convocatorias de los exámenes, desarrollo del Plan de Acción Tutorial, distribución del plan de estudios, horario de clases y calendario de exámenes. Cabría, por tanto, la posibilidad de redefinir las estructuras organizativas del sistema universitario, creando nuevas vías que potencien la participación de los estudiantes en las tomas de decisiones. El alumnado, desde este punto de vista, no sólo se entiende como un mejor aprendiz, sino como un agente educativo de primer orden, miembro de la comunidad educativa, activo, participativo, reflexivo y crítico. 
Centrados en el conocimiento académico del alumnado sobre el Grado, a pesar de valorar de forma positiva el uso de las guías docentes y el conocimiento que dispone sobre las diferentes menciones de especialización, afirman desconocer cuestiones tales como aspectos académicos de su titulación o el nuevo sistema de créditos europeos. El estudiante universitario debe conocer la nueva situación en que se encuentra el sistema universitario gracias al proceso de convergencia europea; no debe permanecer impasible, sumido en el desconocimiento de los cambios producidos sino que, por el contrario, debe definirse como un elemento activo, crítico, capaz de interrogar, cuestionar y establecer propuestas de mejora en pro de una enseñanza universitaria de calidad.

Respecto a la movilidad internacional en el alumnado, se destaca cómo aquellos estudiantes que han desarrollado estancias en centros universitarios europeos muestran unas percepciones más favorables hacia el sistema universitario en cuestiones referidas a la planificación y organización docente así como el conocimiento de que disponen sobre cuestiones académicas del Grado que se encuentran cursando. La participación de los estudiantes en experiencias de este tipo ha podido llevarles a conocer otras realidades, valorando el esfuerzo y la dedicación prestada para la implantación del proceso de convergencia europea en la universidad. Además, el interés del alumnado por cursar parte de sus estudios en otras universidades europeas surge como consecuencia de un mayor compromiso e implicación en sus estudios, lo que puede influir en las percepciones de éstos acerca del sistema universitario.

Los alumnos participantes en el programa Erasmus se muestran más satisfechos con las instalaciones ofrecidas por la institución de Educación Superior, considerando el uso de las guías docentes así como la asistencia y participación a clase, el uso de referencias bibliográficas actuales y de fácil acceso y espacios virtuales elementos favorecedores de un proceso de enseñanza y aprendizaje de calidad. Dichos programas de movilidad europea pretenden que las universidades trabajen desde una perspectiva más internacional, estrechando lazos de cooperación entre el profesorado y alumnado a través de currículos compartidos entre varias instituciones, proyectos de investigación conjuntos, cursos intensivos, elaboración de materiales, dobles titulaciones, etc., lo que les permitirá conocer otros sistemas educativos, otras culturas. 
Por tanto, la enseñanza universitaria debe hacer frente a las modificaciones, demandas, necesidades y expectativas producidas desde los ámbitos sociales, económicos, tecnológicos, políticos, sociales e institucionales. Por ello, y dada estas nuevas situaciones, se precisa conocer las valoraciones de los estudiantes sobre el actual sistema universitario para así establecer propuestas de mejora en aras de una Educación Superior de calidad. Los resultados obtenidos revelan cómo las percepciones de los estudiantes hacia el actual modelo universitario no son muy positivas, lo cual puede deberse a los recientes cambios acontecidos en la Educación Superior gracias al proceso de convergencia europea así como también a la situación socioeconómico en que se desenvuelve el proceso de enseñanza y aprendizaje del alumno en el sistema educativo español.

No obstante, la escasa muestra con la que se ha llevado a cabo dicha investigación no nos permite garantizar la generalización de los resultados a otras muestras. Además, el uso exclusivo del cuestionario como instrumento de recogida de datos puede generar problemas de deseabilidad social y sinceridad, en la que los sujetos tiendan a responder lo que consideran que será evaluado más favorablemente por el investigador, independientemente de sus percepciones. Así pues, de cara a futuras investigaciones, se pretende poder acceder a otras titulaciones para conocer las percepciones de los estudiantes universitarios que cursan otros estudios, estableciendo comparativas entre alumnos de distintos niveles y ámbitos de estudio para así poder valorar las causas que puedan establecer la existencia de diferencias significativas. Además, puede resultar interesante conocer las percepciones de otros agentes educativos sobre el sistema universitario (profesorado, PAS, familias, etc.) y valorar en qué medida las percepciones de los estudiantes se relacionan con las demandas e inquietudes de los otros.

\section{Referencias bibliográficas}

Álvarez Arregui, E., Álvarez García, Mª C., Pascual, M.A., García, M.S. y Pérez, R. (2005). Retos del docente universitario en el contexto de una enseñanza virtual. En Lafuente, $M^{a}$ I. (coord.). ¿Hacia dónde va la educación universitaria americana y europea?: historia, temas y problemas de la universidad: actas del Congreso Internacional. (pp.489-495). León: Universidad de León. Servicio de Publicaciones.

Bracht, O., Engel, C., Janson, K., Over, A., Schomburg, H y Teichler, U. (2006). The 
Percepciones de los estudiantes sobre el sistema universitario en el marco del Espacio Europeo de Educación Superior: participación en programas de movilidad internacional M. ${ }^{a}$ del Carmen Pegalajar Palomino, Eufrasio Pérez Navío y M. ${ }^{a}$ Jesús Colmenero Ruiz

Proffessional value of Erasmus mobility. Internacional Centre for Higuer Education Research, Kassel. Disponible en: http://ec.europa.eu/education/erasmus/doc/publ/ evalcareer.pdf (consultado el 17 de Octubre de 2013).

Bracht, O., Engel, K., Janson, A., Over, Schomburg, H. y Teichler, U. (2006). The professional value of ERASMUS Mobility, Final Report. International Centre for Higuer Education Research (INCHER-Kassel). Kassel: University of Kassel.

Brakel, R.L. van; Huisman, J.; Luijten- Lub, A.; Maussen, M. y Van der Wende, M.C. (2004). External evaluation of Erasmus institutional and national impact. La Haya: CHEPS-Price Waterhouse Coopers.

Carrera, M.; Bonete, R. y Muñoz, R. (2007). El programa Erasmus en el marco del valor económico de la Enseñanza del Español como Lengua Extranjera. Disponible en: http://www.researchgate.net/publication/28246297_El_programa_ERASMUS_en_el_ marco_del_valor_econmico_de_la_Enseanza_del_Espaol_como_Lengua_Extranjera/ file/79e4151363b3b9fc9b.pdf (consultado el 1 de mayo de 2013).

Castaño, E., Benito, A., Portela, A. y Rodríguez, R.M. (2007). Repercusiones en los alumnos de primer curso de la implantación del Espacio Europeo. Revista complutense de Educación, 18(1), 199-216.

Coterón, J., Franco, E. y Gil, J. (2012). Opinión del alumnado sobre la implantación del Espacio Europeo de Educación Superior en estudios de Ciencias de la Actividad Física y del Deporte. Revista Complutense de Educación, 23(1), 191-206.

Edwards, M., Donderis, V. y Ballester, E. (2005). La participación del profesorado y de los estudiantes: factor clave para el éxito del proceso de convergencia. Actas del XIII Congreso Universitario de Innovación Educativa en las Enseñanzas Técnicas. Maspalomas: Gran Canaria.

Escorcia, R.E., Gutiérrez, A.V. y Henríquez, H.J. (2007). La educación superior frente a las tendencias sociales del contexto. Educación y Educadores, 10(1), 63-77.

ESU (2010). StudentCentredLearning: Time for a New Paradigm in Education. European Students'Union.

Figel, J. (2006). My vision for European Student Mobility in the next Decade. The future of European student mobility. Conference organized by the UK Erasmus Student Committee. Bruselas.

Font-Mayolas, S. y Masferrer, L. (2010). Conocimientos y actitudes de estudiantes universitarios respecto al Espacio Europeo de Educación Superior. Revista de Formación e Innovación Educativa Universitaria, 3(2), 88-96.

Froment, E., Kolher, J., Purser, L, Wilso, L., Davies, H. y Schurings, G. (2010). EUA Bologna Handbook. Making Bologna Work. Berlin:EUA.

Gairín, J., Feixas, M., Guillamón, C., Quinquer, D. (2004). La tutoría académica en el escenario europeo de Educación Superior. Revista Interuniversitaria de Formación del Profesorado, 18, 66-77.

García, J.V. y Pérez, Mª C. (2008). Espacio Europeo de Educación Superior, competencias profesionales y empleabilidad. Revista Iberoamericana de Educación, 46(9), 1-12.

Gil, J. (2012). La evaluación del aprendizaje en la universidad según la experiencia de los estudiantes. Estudios sobre educación, 22, 133-153.

Herrera, L, Lorenzo, O. y Rodríguez, C. (2008). Las tutorías en el Espacio Europeo de 
Percepciones de los estudiantes sobre el sistema universitario en el marco del Espacio Europeo de Educación Superior: participación en programas de movilidad internacional M. a del Carmen Pegalajar Palomino, Eufrasio Pérez Navío y M. ${ }^{a}$ Jesús Colmenero Ruiz

Educación Superior: valoración de su implementación en la titulación de Maestro. Revista de Investigación Psicoeducativa, 6(1), 65-85.

Herrera, L. (2007). Experiencia Piloto de Implantación del Sistema de Transferencia de Créditos Europeos (ECTS) en la Titulación de Maestro. Valoración del profesorado y el alumnado participante. En R. Roig (Dir.). Investigar el cambio curricular en el Espacio Europeo de Educación Superior (pp. 159-178). Alicante: Editorial Marfil.

Herrera, L. y Cabo, J.M. (2008). Experiencias piloto de implantación del sistema europeo de transferencia y acumulación de créditos (ECTS). Reflexiones derivadas de su aplicación práctica en diferentes universidades españolas. Granada: Editorial Comares.

Herrera, L. y Enrique, D. (2008). Proyectos de Innovación en tutorías en la Universidad de Granada: Análisis de los instrumentos empleados. Profesorado. Revista de Currículum y Formación del Profesorado, 12(2), 1-18.

INE (2012). El programa Erasmus. Disponible en http://ine.es (consultado el 01 de mayo de 2013).

Jacobs, B y Van Der Ploeg, F. (2006). Guide to reform of higher education: a European perspectiva. Economic Policy, 21(47), 535-592.

Leo, M. y Cubo, S. (2012). El Espacio Europeo de Educación Superior. Actitudes del alumnado de la Universidad de Extremadura. Campo Abierto, 31(1), 29-50.

Méndez, C. (2005). La implantación del sistema de créditos europeo como una oportunidad para la innovación y mejora de los procedimientos de enseñanza aprendizaje en la universidad. Revista Española de Pedagogía, 63(230), 43-62.

Méndez, R.M. (2008). Los jóvenes universitarios y su (des)orientación ante los nuevos retos que plantea el EEES. Educatio Siglo XXI, 26, 197-224.

Michavila, F. y Esteve, F. (2011). La Ilegada a la universidad: ¿oportunidad o amenaza? Participación educativa, 17,69-85.

Moreno, S., Bajo, M.T., Moya, M., Maldonado, A., Tudela, P. (2007). Las competencias en el nuevo paradigma educativo para Europa. Granada: Vicerrectorado de Planificación, Calidad y Evaluación Docente de la Universidad de Granada.

Otero, $M^{a}$ C., Ferro, C. y Vila, M. (2012). Satisfacción del alumnado ante la implantación del modelo del EEES. Análisis comparativo. Hekademos: revista educativa digital, 12, 35-42.

Pineda, P., Moreno, M.V. y Belvis, E. (2008). La movilidad de los universitarios en España: estudio sobre la participación en los programas Erasmus y Sicue. Revista de Educación, 346, 363-399.

Rodríguez, R. (2009). El reto de la convergencia europea: necesidades y cambios. Revista de Formación e Innovación Educativa Universitaria, 2(1), 20-30.

Sander, P. (2005). La investigación sobre nuestros alumnos en pro de una mayor eficacia en la enseñanza universitaria. Electronic Journal of Research in Educational Psychology, 3(1), 113-130.

Sevillano, M.L. (2004). Didáctica en el siglo XXI. Ejes en el aprendizaje de calidad. Madrid: McGrawHill.

Tempus Public Foundation (2006) Dissemination of results and best practices for raising the profile of Erasmus Mobility. Tempus Public Foundation. Dublín.

Tójar, J.C. y Matas, A. (2009). Fundamentos metodológicos básicos. En A. Pantoja (coord.). Manual básico para la realización de tesinas, tesis y trabajos de investigación. Madrid: EOS. 
Percepciones de los estudiantes sobre el sistema universitario en el marco del Espacio Europeo de Educación Superior: participación en programas de movilidad internacional M. ${ }^{a}$ del Carmen Pegalajar Palomino, Eufrasio Pérez Navío y M. ${ }^{a}$ Jesús Colmenero Ruiz

Tomusk, V. (2006). Creating the European Area of Higuer Education: Voices from the periphery. Dordrecht: Springer.

Villar, L.M. y De La Rosa, O.M. (2004). Manual para la excelencia en la enseñanza superior. Madrid: Pearson. 